\title{
Les multiples facettes de la cytokine HILDA/LIF
}

Yannick Jacques

Ignacio Anegon Marie-Martine Hallet Anne Godard Jean-Paul Moisan Jean-Paul Soulillou

\section{ADRESSE}

Y. Jacques: directeur de recherche au Cnrs. I. Anegon : chargé de recherche à l'Inserm. M.M. Hallet : maître de conférence des universités. A. Godard : maître de conférence des universités, praticien hospitalier. J.-P. Moisan : maître de conférence des universités, praticien hospitalier. J.P. Soulillou : professeur d'immunologie, directeur de l'U.211 de l'Inserm. Inserm U.211, institut de biologie, 9, quai Moncousu, 44035 Nantes Cedex, France.

La cytokine HILDA/LIF a été caractérisée de manière indépendante par plusieurs équipes et sur la base d'activités biologiques diverses allant de l'induction à la différenciation de certaines cellules leucémiques à la stimulation de la prolifération d'autres ou à l'inhibition de la différenciation des cellules souches embryonnaires. L'HILDA/LIF se fixe à un récepteur composé de deux ou trois types de sous-unités, une ou deux d'entre elles étant partagées avec d'autres cytokines : l'oncostatine, l'interleukine 6 et le facteur neurotrophique ciliaire (CNTF). Cela explique probablement que l'élimination totale de toute synthèse de HILDA/LIF par recombinaison homologue n'entraîne que peu de conséquences (uniquement une impossibilité à la nidation de l'embryon chez la femelle homozygote déficiente) alors que l'hyperproduction de cette même cytokine a des effets pléiotropiques.

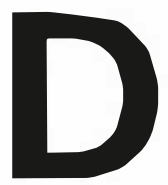

e nombreux acteurs cellulaires coopèrent pour assurer un développement coordonné des réactions immunitaires. Cette orchestration est essentiellement assurée par des facteurs solubles sécrétés par ces cellules et qui agissent localement ou à distance et à de très faibles concentrations par le biais de récepteurs spécifiques. Ces facteurs sont regroupés sous le nom générique de cytokines. Pour la plupart d'entre elles, un nombre limité de sites cellulaires de production et d'action ont été décrits (c'est le cas de l'interleukine 2). Pour certaines, cependant, il devient de plus en plus clair que leurs spectres d'action dépassent largement le cadre du système immunitaire. L'HILDA/LIF est l'exemple même de ce type de cytokine pléïo- trope et sa dénomination comme LIF ou HILDA paraît aujourd'hui très restrictive. Bien qu'identifiée originellement dans le système hématopoiétique par son effet inducteur de la différenciation de la lignée leucémique myéloïde M1 (activité LIF) [1] et sa capacité à induire la prolifération de la lignée murine DA1a (activité HILDA) [2], il s'avère que l'HILDA/LIF exerce de nombreuses activités en dehors de ce système (Tableau I), nombre d'entre elles étant par ailleurs partagées avec d'autres cytokines comme l'IL-6, l'oncostatine M (OSM), le G-CSF, le facteur neurotrope ciliaire (CNTF). Parmi ces multiples activités, celles qui semblent actuellement les plus intéressantes d'un point de vue biologique, et qui pour l'instant s'avèrent spécifiques de l'HILDA/LIF, sont d'une 


\section{RÉFÉRENCES}

1. Tomida M, Yamamoto-Yamagushi T, Homuzi M. Purification of a factor inducing the differentiation of mouse myeloid leukemia cells from conditionned medium of fibroblasts 929 cells. $J$ Biol Chem $1984 ; 259$ : 10978-82.

2. Moreau JF, Bonneville M, Peyrat MA, et al. Capacity of alloreactive human $\mathrm{T}$ cell clones to produce a factor inducing proliferation of the IL-3-sensitive DA1 murine cell line. Evidence that this production is under IL-2 control. Ann Immunol Inst Pasteur 1986 ; 137 c : $25-37$

3. Hilton DJ. LIF : lots of interesting functions. Trends Biochem Sci 1992; 17 : 72-6.

4. Smith AG, Nichols J, Robertson M, et al. Differentiation Inhibitory Activity (DIA/LIF) and mouse development. Dev Biol $1992 ; 151: 339-51$

5. Rose TM, Bruce AG. Oncostatin M is a member of a cytokine family that includes leukemia-inhibitory factor, granulocyte colony-stimulating factor, and interleukin 6 . Proc Natl Acad Sci USA 1991; 88 : 8641-5.

6. Stahl J, Gearing DP, Willson TA, et al. Structural organisation of the genes for murine and human lcukemia inhibitory factor. J Biol Chem 1990; 265 : 8833-41.

7. Rathjen PD, Toth S, Willis A, et al. Differentiation inhibiting activity is produced in matrix-associated and diffusible forms that are generated by alternate promotor usage. Cell $1990 ; 62: 1105-14$

8. Anegon I, Moreau JF, Godard A, et al. Production of human interleukin for DA cells HILDA/Leukemia Inhibitory Factor LIF by activated monocytes. Cell Immunol $1990 ; 130: 50-65$

9. Bentouimou N, Moreau JF, Peyrat MA et al. Effects of cytokine A on HILDA/LIF gene expression in human $\mathrm{T}$ cells. Transplantation 1992 (sous presse).

10. Sutherland GR, Baker E, Hyland VJ, et al. The gene for leukemia inhibitory factor (LIF) maps to 22q12. Leukemia 1989 ; 3 : $9-13$.

11. Kola I, Davey A, Gough NM. Localization of the murine leukemia inhibitory factor gene near the centromere on chromosome 11. Growth Factors 1990 ; 2 : 235-40.

12. Godard A, Heymann D, Raher S, et al. High and Low affinity receptors for human interleukin for DA cells/leukemia inhibitory factor on human cells. Molecular characterization and cellular distribution. J Biol Chem 1992 ; 267 : 3214-22. part sa capacité à maintenir la pluripotentialité des cellules souches embryonnaires in vitro, d'autre part son rôle majeur dans les processus d'implantation utérine de l'embryon. Après avoir présenté les caractéristiques biochimiques et moléculaires de l'HILDA/LIF et de ses récepteurs, cet article tentera de faire le point sur ses différentes propriétés biologiques in vitro et in vivo. Les analogies structurales et les redondances fonctionnelles au sein de la famille HILDA/LIF, IL-6, OSM, G-CSF, CNTF seron également soulignées. Les lecteurs intéressés sont par ailleurs invités à se référer aux excellentes revues récentes parues sur l'HILDA/LIF [3, 4].

\section{Structure protéique}

L'HILDA/LIF est une glycoprotéine monomérique dont la masse moléculaire est très variable selon les sources cellulaires de production (de 38 à $67 \mathrm{kDa}$ ) (Tableau II et figure 1). La grande majorité de cette hétérogénéité réside dans son degré variable de glycosylation. Pour l'HILDA/LIF purifiée à partir de la lignée leucémique HSB2 (43 kDa), les maillons $\mathrm{N}$ glycosylés représentent environ $50 \%$ de la masse moléculaire $(21 \mathrm{kDa})$ tandis que les maillons O-glycosylés y contribuent pour 2 à $3 \mathrm{kDa}$.

Les ADNc codant pour l'HILDA/ LIF de souris, de rat et humain prédisent une séquence codante de 202 acides aminés. Les 22 premiers résidus forment le peptide signal tandis que la protéine mûre est composée de 180 acides aminés restants avec une masse moléculaire (squelette polypeptidique) de $23 \mathrm{kDa}$. Les homologies inter-espèces de séquence primaire sont très fortes : $79 \%$ entre homme et souris, $92 \%$ entre rat et souris et $82 \%$ entre rat et homme La molécule HILDA/LIF contient sept (rat) ou six (homme, souris) cystéines dont l'engagement dans des ponts disulfures est nécessaire à l'activité biologique. Les sites potentiels, de $\mathrm{N}$-glycosylation sont au nombre de sept à huit, dont quatre sont fonctionnels (positions 9, 34, 96 et 116). Le rôle de la glycosylation importante de la molécule n'est pour l'instant pas élucidé. Des formes déglycosylées enzymatiquement ou recombinantes non glycosylées (E. coli) montrent des activités biologiques spécifiques inchangées par rapport aux formes natives glycosylées.

Des études de prédiction de structure secondaire suggèrent que l'HILDA/ LIF contient quatre domaines structurés en hélices $\alpha$ (figure 1). Ces domaines, organisés de manière antiparallèle, pourraient adopter une structure tertiaire en faisceau, semblable à celle établie par cristallographie pour l'hormone de croissance (GII), et dans laquelle la face exposée de l'hélice $\alpha$ C-terminale (hélice D) constituerait un site de liaison au récepteur. Un tel type de structure semble être prédit pour de nombreuses cytokines dont l'IL-6, l'OSM et le GCSF, facteurs qui, comme l'HILDA/LIF, induisent la différentiation de la lignée M1. Des homologies de séquence primaire existent également entre ces quatres cytokines, la plus significative $(30 \%)$ étant entre HILDA/LIF et OSM [5].

\section{Gènes : structure, transcription et localisation chromosomique}

Les gènes humain et murin codant pour l'HILDA/LIF ont des tailles respectives de 7,6 et 8,7 kilopaires de bases et sont composés de trois exons et deux introns [6] (figure 2). En dehors des régions codantes très homologues entre deux espèces, certaines séquences des régions non codantes sont hautement conservées (intron I ; > $75 \%$ d'homologie) et pourraient correspondre à des éléments de contrôle en cis de l'expression du gène. Il y a $90 \%$ d'homologie entre les régions 5' non transcrites et 5' non traduites des gènes humain et murin (région d'homologic 1 de la figure 1). Ce type d'observation a déjà été fait pour d'autres gènes, comme celui du GM-CSF où ces séquences sont impliquées dans les régulations spécifiques de tissu. Les gènes humain et murin ont une région 3' non traduite exceptionnellement longue de 3200 paires de bases. La fonction de ces grandes régions, retrouvées également dans les gènes des récepteurs d'hormones stéroïdes et du bFGF, n'est pas connue. L'homologie la plus importante se trouve dans la région 7 (figure 1) qui 


\begin{tabular}{|c|c|c|c|c|}
\hline \multicolumn{5}{|c|}{ Tableau I } \\
\hline Acronyme & Nom & Cible & Effet & Références \\
\hline HILDA & Human interleukin for DA cells & Lignée DA1a & Induction prolifération & {$[2,23]$} \\
\hline LIF & Leukemia inhibitory factor & Lignée $\mathrm{M} 1$ & Inhibition prolifération & [24] \\
\hline D-factor & Differentiation factor & Lignée M1 & Induction différenciation & [1] \\
\hline & Differentiation inducing factor & Lignée M1 & Induction phagocytose & [42] \\
\hline CNDF & $\begin{array}{l}\text { Cholinergic neuronal } \\
\text { Differentiation factor }\end{array}$ & $\begin{array}{l}\text { Neurones } \\
\text { Sympathiques }\end{array}$ & Induction acétylcholine & {$[40]$} \\
\hline HSF III & Hepatocyte stimulating factor III & Hépatocytes & Induction protéines phase aiguë & [41] \\
\hline DRF & Differentiation retarding factor & $\begin{array}{l}\text { Cellules souches } \\
\text { embryonnaires }\end{array}$ & Inhibition différenciation & [37] \\
\hline DIA & Differentiation inhibitory activity & $\begin{array}{l}\text { Cellules souches } \\
\text { embryonnaires }\end{array}$ & Inhibition différenciation & {$[36]$} \\
\hline OAF & Osteoclast activating factor & Tissu calvaire & Relargage de calcium & {$[42]$} \\
\hline MLPLI & $\begin{array}{l}\text { Melanoma derived lipoprotein } \\
\text { lipase inhibitor }\end{array}$ & Lignée 3T3-Lli & Inhibition lipoprotéine lipase & {$[26]$} \\
\hline & & Myoblastes & Induction prolifération & [46] \\
\hline
\end{tabular}

contient des séquences impliquées dans la formation de l'extrémité 3' de l'ARN (séquences riches en GT) et dans sa stabilité (séquences riches en AT). Deux sites d'initiation de la transcription, situés à 100 paires de bases l'une de l'autre, ont été mis en évidence. Ils sont tout deux précédés de séquences consensus TATA box. Enfin, les régions promotrices des gènes humain et murin contiennent des régions régulatrices riches en bases pyrimidiques, ainsi que des sites potentiels de liaison pour des protéines nucléaires régulatrices (type AP-2 et SP-1).

Une forme diffusible (D) et une forme associée à la matrice extracellulaire (M) de l'HILDA/LIF murine ont été décrites [7]. Leur localisation semble être déterminée par un épissage alternatif du messager (figure 2) ; les sept premiers acides aminés du peptide signal de la forme $\mathrm{D}$, codés par le premier exon, sont remplacés dans la forme $\mathrm{M}$ par quatre autres acides aminés. La séquence de ces derniers serait déterminée par un petit exon supplémentaire situé à l'intérieur du premier intron (E1). Du fait de l'utilisation de promoteurs différents (Pr, Pr'), l'expression des formes $\mathrm{D}$ et $\mathrm{M}$ peut être contrôlée de manière indépendante pendant la différenciation cellulaire et il est probable que l'une ou l'autre de ces formes prédomine suivant les tissus et les cellules où la molécule est exprimée.

$\mathrm{m} / \mathrm{s} n^{\circ} 9$ vol. 8 , novembre 92

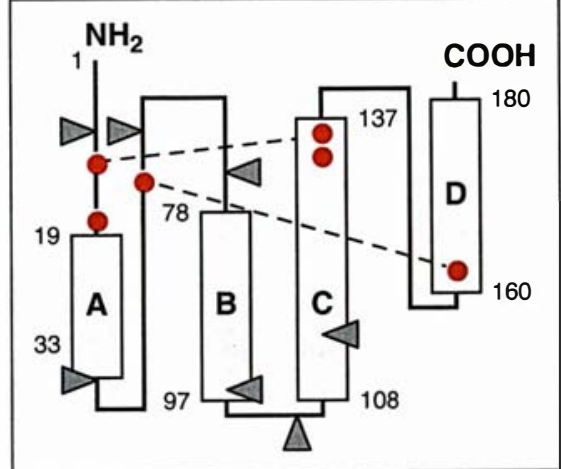

Figure 1. Modèle prédictif de la structure secondaire de I'HILDA/LIF humain. Traits pleins : squelette polypeptidique avec numérotation des résidus. Rectangles A B C $D$ : domaines structurés en hélices $\alpha$. Triangles : sites potentiels de $\mathrm{N}$-glycosylation. Points rouges: résidus cystéines. Traits discontinus : localisation hypothétique des ponts disulfures.

Les mécanismes de contrôle de la transcription du gène HILDA/LIF sont pour l'instant très peu documentés. Des expériences de blocage de la traduction des messagers HILDA/LIF ont montré que lors d'une stimulation des monocytes par des mitogènes, la mise en route de la transcription d'un gène requiert la synthèse d'une (ou de) protéines relais [8]. Dans les lymphocytes $\mathrm{T}$ activés, l'accumulation des transcrits et la sécrétion de la protéine sont en grande partie bloqués par la ciclosporine $\mathrm{A}$ [9].
Le gène de l'HILDA/LIF a été localisé chez l'homme sur le chromosome 22 en q12-1 [10], dans une région distale par rapport au point de cassure impliqué dans le sarcome d'Ewing. Cette position sur le génome humain en fait un marqueur génétique intéressant pour l'étude du locus impliqué dans la neurofibromatose de type (NF2) puisque le gène HILDA/LIF est situé entre deux marqueurs génétiques D22S1 et D22S28 flanquant ce locus. Aucun polymorphisme de restriction du gène HILDA/LIF n'a été constaté à l'heure actuelle.

Toutefois, des études en cours sur les régions flanquant ce gène devraient permettre de définir un ou des marqueurs génétiques dans cette région. Le gène homologue murin a été localisé près du centromère du chromosome 11 (bande A1-A2), dans une région de synthénie avec le chromosome 22 humain [11].

Le gène humain de l'OSM est organisé de manière tout à fait similaire à celui de l'HILDA/LIF (trois exons de taille similaire, deux introns, une séquence signal située à cheval sur les deux premiers exons) [5]. Il est également localisé sur le chromosome 22. Les analogies de structure primaire et secondaire, d'organisation et de localisation génique sont autant d'éléments qui plaident en faveur d'une origine ancestrale commune pour les deux gènes. 


\section{Récepteurs}

De nombreux types cellulaires expriment des récepteurs membranaires de haute affinité pour l'HILDA/LIF. Chez la souris, c'est le cas de la lignée leucémique myéloïde M1 et de la lignée DA1a. Ces récepteurs sont présents à de faibles densités (20 à 100 sites/cellule) et lient la cytokine avec une haute affinité $\left(\mathrm{K}_{\mathrm{D}}=50\right.$ $\left.\times 10^{-12} \mathrm{M}\right)$. Des analyses par autoradiographie ont indiqué la fixation d'HILDA/LIF par les monocytes, les macrophages et leurs précurseurs dans la moelle osseuse, la rate et la cavité péritonéale. Aucune fixation n'est observée sur les neutrophiles, les éosinophiles, les mastocytes, les érythrocytes ainsi que les cellules lymphocytaires. Des récepteurs de haute affinité ont été identifiés sur des cellules souches embryonnaires (ES) et des lignées de carcinomes embryonnaires (EC), sur des ostéoblastes murins et de rat mais pas sur les ostéoclastes, sur des hépatocytes murins fotaux et adultes, enfin sur une lignée épithéliale de rein de porc. Chez l'homme, des récepteurs de haute affinité pour l'HILDA/LIF sont exprimés par une panoplie de types cellulaires [12]. Parmi les cellules du sang périphérique humain, seuls les monocytes sont positifs (20 sites/cellule), à l'inverse des cellules $\mathrm{NK}$, des polynucléaires, des plaquettes et des lymphocytes. Des clones lymphocytaires $\mathrm{T}$ alloréactifs sont également négatifs. Parmi les lignées tumorales testées, des lignées myélomonocytaires (U937, THP1) et lymphocytaires $\mathrm{T}$ (HSB2) ou $\mathrm{B}(\mathrm{DAB})$ se révèlent négatives. En revanche, de nombreuses lignées tumorales d'origine non hématopoïétique expriment des récepteurs de haute affinité. C'est le cas des mélanomes (FOSS, C32) et de différents carcinomes (côlon : SW948 ; sein : HBL100 ; placenta : JAR, JEG).

Plusieurs types cellulaires dont les monocytes, les ostéoblastes et certaines lignées tumorales non hématopoïétiques (SW948, FOSS, C32) expriment à la fois le récepteur et la lymphokine, ce qui suggère que l'HILDA/LIF pourrait exercer une partie de ses actions par des mécanismes de type autocrine.

De nombreuses cellules humaines expriment également une composante de liaison de basse affinité pour l'HILDA/LIF [12]. Sur la cellule JAR (choriocarcinome humain), ces récepteurs sont sept fois plus nombreux que les sites de haute affinité (4 400 sites/cellules contre 600) et sont caractérisés par une constante de dissociation à l'équilibre $\left(\mathrm{K}_{\mathrm{D}}=4\right.$ $\times 10^{-9} \mathrm{M}$ ) environ 100 fois plus forte que celle caractérisant les récepteurs de haute affinité. Cette différence d'affinité se traduit sur le plan cinétique par une constante d'association 30 fois plus faible et une constante de dissociation 20 fois plus forte que l'HILDA/LIF sur les sites de basse affinité par rapport à ceux de haute affinité. Des expériences de pontage covalent ont montré l'association de l'HILDA/LIF à deux chaînes majeurs de masse moléculaire 250 kDa et 120 kDa. La chaîne p250 constituerait le récepteur de basse affinité alors que la forme de haute affinité serait formée de l'association non covalente des deux chaînes.

L'ADNc d'un premier récepteur de l'HILDA/LIF a été récemment cloné [13]. L'ADNc obtenu code pour une protéine de 1097 acides aminés comprenant à son extrémité $\mathrm{N}$-terminale une séquence signal de 44 résidus et des domaines extracellulaire, transmembranaire et cytoplasmique de 789, 26 et 238 résidus. Lorsqu'il est exprimé dans des cellules COS-7, ce récepteur a une masse moléculaire de $190 \mathrm{kDa}$ et fixe l'HILDA/LIF avec un $K_{D}$ correspondant aux formes de faible affinité. Un clone ADNc murin a également été obtenu par hybridation avec une sonde du récepteur humain et code pour un récepteur soluble (719 acides aminés) présentant $76 \%$ d'homologie avec le domaine extracellulaire de l'homologue humain.

Un autre $\mathrm{ADNc}$ codant pour une chaîne permettant la conversion de la liaison HILDA/LIF basse affinité en haute affinité a également été obtenu [14]. L'analyse de sa séquence montre qu'il code pour une protéine identique à la chaîne gp130 du récepteur de l'interleukine 6 (IL-6). Les sousunités gp190 et gp130 font toutes deux partie de la superfamille des récepteurs hématopoïétiques $[15,16]$. Le domaine principal d'homologie au sein de cette famille (CHR, cytokine 


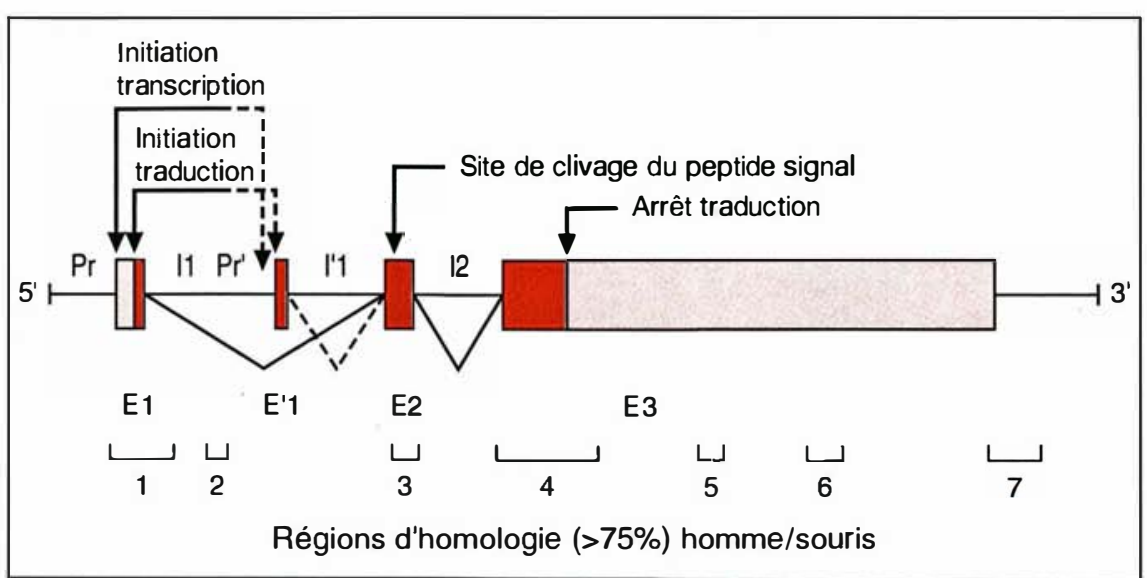

Figure 2. Organisation générale des gènes humain et murin de I'HILDA/LIF et localisation des zones d'homologie. $\mathrm{Pr}^{\prime} \mathrm{Pr}^{\prime}$ : régions promotrices. E: exons. I: introns. (Adapté d'après [6].)

receptor homologous) (figure 3) se situe dans la partie extracellulaire impliquée dans la liaison du ligand. Il a une taille d'environ 210 acides aminés comprenant quatre résidus cystéines conservés dans sa moitié $\mathrm{N}$ terminale et un motif conservé TrypSer-X-Tryp-Ser (WSXWS) à son extrémité C-terminale. Il est retrouvé dans les récepteurs de l'IL-2 (chaîne $\beta$ ), IL-3, IL-4, IL-5, IL-6, IL-7, G-CSF, GM-CSF, érythropoïétine, hormone de croissance $(\mathrm{GH})$, prolactine (PRL) et du facteur neurotrophique ciliaire (CNTF).

Ces domaines CHR contiennent une duplication structurale interne en deux régions d'environ 100 acides aminés. Des analyses prédictives indiquent que chacune de ces régions est

composée de sept feuillets $\beta$ organisés de manière antiparallèle pour former une structure de type barillet semblable à celle décrite pour les domaines constants des immunoglobulines [17]. L'arrangement en $\mathrm{V}$ de ces deux régions pourrait former une gorge constituant le site de liaison de la cytokine. Certains récepteurs de la famille hématopoiétique comme la gp130 et la gp190 comprennent également des domaines structuraux de type immunoglobuline (Ig) et/ou de type contactine (succession de trois modules fibronectines type III ; FNIII) (figure 3).

Au-delà de cette analogie structurale, les membres de cette famille de récepteurs hématopoïétiques manifestent des similarités fonctionnelles de
Tableau II

\section{SOURCES CELLULAIRES D'HILDA/LIF}

\begin{tabular}{|c|c|c|c|c|}
\hline & Homme & Souris & Rat & Bovin \\
\hline Lymphocytes $T$ activés & + & + & & \\
\hline Splénocytes activés & & + & & \\
\hline Monocytes activés & + & & & \\
\hline Macrophages & & + & & \\
\hline Neutrophiles & & + & & \\
\hline Cellules stromales moelle osseuse & + & & & \\
\hline Cellules souches embryonnaires & & + & & \\
\hline Tissus extra-embryonnaires & & + & & \\
\hline Ostéoblastes & + & + & + & \\
\hline Cellules épithéliales thymiques & + & & & \\
\hline Fibroblastes & & + & & \\
\hline Cellules myocardiques & & & + & \\
\hline Hépatocytes & & & + & \\
\hline Cellules hypophysaires & & & & + \\
\hline
\end{tabular}

$\mathrm{m} / \mathrm{s} n^{\circ} 9$ vol. 8 , novembre 92 plus en plus frappantes. Le premier exemple concerne les récepteurs de l'IL-3, de l'IL-5 et du GM-CSF (pour revue voir $[16,18]$ ). Les chaînes $\alpha$ de ces récepteurs sont distinctes et responsables de la liaison spécifiques des cytokines respectives. Elles lient cependant le ligand avec une basse affinité et n'induisent pas d'effet fonctionnel. Pour former des structures fonctionnelles de haute affinité, ces récepteurs utilisent une chaîne commune $\beta$ (clonée sous le nom de KII97) en association avec les chaînes $\alpha$ respectives. Cette chaîne $\beta$, qui toute seule n'est capable de lier aucune des cytokines, agit comme un convertisseur d'affinité commun aux trois récepteurs et est nécessaire à l'initiation de la transduction du signal biologique. La mise en évidence de cet élément commun explique au moins en partie la similitude des activités biologiques exercées par ces trois cytokines sur certains types de cellules comme les éosinophiles.

Un mécanisme du même type s'avère présider à la formation des structures fonctionnelles des récepteurs de l'HILDA/LIF, de l'IL-6, de l'OSM et du CNTF. Suite à la découverte de la chaîne gp130 comme élément convertisseur commun aux chaînes gp190 (HILDA/LIF) et gp80 (IL-6), il a été montré que la gp130 était également un récepteur de basse affinité pour l'OSM. De plus l'OSM utilise, tout comme l'HILDA/LIF, les chaînes gp130 et gp190 en association pour se lier avec une haute affinité ([4], $m / s n^{\circ} 5$, vol. 8, p. 490). La chaîne $\alpha$ du récepteur du CNTF (gp72) a une forte homologie avec la chaîne gp80 (IL-6) et est ancrée à la membrane par le biais d'une liaison glycosyl-phosphatidyl-inositol (GPI) [19]. Des travaux très récents ont montré que la structure fonctionnelle du récepteur du CNTF implique la participation de la chaîne gp130 et suggèrent également l'association de la chaîne gp190 à cette structure [20]. Un modèle (figure 3 ) semble ainsi se dessiner dans lequel la chaîne gp130 serait un élément commun de transduction associé aux récepteurs des quatre cytokines, IL-6, HILDA/LIF, de l'OSM et du CNTF. La chaîne gp190 serait également un deuxième élément de transduction commun aux 


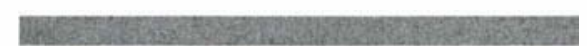

\section{RÉFÉRENCES}

25. Gascan H, Anegon I, Praloran V, et al. Constitutive production of human Interleukin for DA cells/Leukemia Inhibitory Factor by human tumor cell lines derived from various tissues. J Immunol $1990 ; 144$ : 2592-8.

26. Mori MK, Yamaguchi K, Abe K. Purification of a lipoprotein lipase-inhibiting protein produced by a melanoma cell line associated with cancer cachexia. Biochem Biophys Res Commun 1989 ; 160 : 1085-92.

27. Hilton DJ, Nicola NA, Waring PM, et al. Clearance and fate of leukemia-inhibitory factor (LIF) after injection into mice. $J$ Cell Physiol 1991; 148 : 430-9.

28. Bhatt H, Brunet LJ, Steward CL. Uterine expression of Leukemia Inhibitory Factor coincides with the onset of blastocyst implantation. Proc Natl Acad Sci USA 1991 ; 88 : 11408-12.

29. Conquet F, Brulet P. Developmental expression of myeloid leukemia inhibitory factor gene in preimplantation blastocysts and in extraembryonic tissue of mouse embryos. Mol Cell Biol 1990 ; 10 : 3801-5.

30. Moreau JF, Bonneville M, Godard A, et al. Characterisation of a factor produced by human $\mathrm{T}$ cell clones exhibiting eosiniphilactivating and burst-promoting activities. $J$ Immunol 1987 ; 138 : 3844-9.

31. Leary AG, Wong GG, Clark SC, et al. Leukemia inhibitory factor differentiationinhibiting activity/human interleukin for DA cells augments proliferation of human hematopoietic stem cells. Blood $1990 ; 75: 1960-4$.

32. Godard A, Gascan H, Naulet J, et al. Biochemical characterization and purification of HILDA, a human lymphokine active on eosinophils and bone marrow cells. Blood $1988 ; 71$ : 1618-23

33. Fletcher FA, Williams DE, Maliszewski C, et al. Murine leukemia inhibitory factor enhances retroviral vector infection efficiency of hematopoietic progenitors. Blood $1990 ; 76: 1098-103$.

34. Metcalf D, Nicola NA, Gearing DP. Effects of injected leukemia inhibitory factor on hematopoietic and other tissues in mice. Blood 1990 ; 76 : 50-6.

35. Metcalf D, Gearing DP. Fatal syndrome in mice engrafted with cell producing high levels of the leukemia inhibitory factor. Proc Natl Acad Sci USA 1989 ;

\begin{tabular}{|c|c|c|}
\hline \multicolumn{3}{|c|}{$\begin{array}{c}\text { Tableau III } \\
\text { CARACTÉRISTIQUES DE L'HILDA/LIF }\end{array}$} \\
\hline & Homme & Souris \\
\hline Chromosome & 22 q $12-1$ & $11 \mathrm{~A} 1-\mathrm{A} 2$ \\
\hline Taille du gène (kilobases) & 7,6 & 8,7 \\
\hline Introns/exons & $2 / 3$ & $2 / 3$ \\
\hline Taille des messagers (kilobases) & 4,2 et 1,8 & 4,2 \\
\hline \multicolumn{3}{|l|}{ Précurseur (acides aminés) } \\
\hline Protéine diffusible (D) & 202 & 202 \\
\hline Protéine matricielle (M) & $?$ & 199 \\
\hline \multicolumn{3}{|l|}{ Protéine mature (acides aminés) } \\
\hline Protéine diffusible & 180 & 180 \\
\hline Protéine matricielle & $?$ & 180 \\
\hline Sites de N-glycosylation & 7 & 7 \\
\hline Groupements sulfhydriles & 6 & 6 \\
\hline
\end{tabular}

récepteurs de l'HILDA/LIF, OSM et $\mathrm{CNTF}$, tandis que le récepteur de l'IL-6 pourrait, par souci de symétrie, faire intervenir deux chaînes gp130. Pour ces récepteurs, l'élément conférant la spécificité de reconnaissance du ligand serait une chaîne $\alpha$, plus courte que les éléments communs de transduction, et elle-même dépourvue d'activité de transduction. Cette chaîne $\alpha$ est identifiée pour les récepteurs de l'IL-6 (gp80) et du CNTF (gp72). Elle reste à être identifiée pour les récepteurs du LIF et de l'OSM.

Ce modèle permet de donner une base moléculaire à la fois à la redondance fonctionnelle de ces cytokines et à leurs pléiotropies. L'utilisation par ces différentes cytokines d'une machinerie de transduction commune permet, d'une part, d'expliquer qu'elles aient des activités semblables sur de nombreux types cellulaires comme la lignée M1 (HILDA/LIF, OSM et IL-6), les hépatocytes et les myoblastes (HILDA/LIF et IL-6) ou les cellules nerveuses (HILDA/LIF et CNTF). L'expression tissulaire plus (CNTF) ou moins (HILDA/LIF, IL-6, OSM) restreinte des chaînes $\alpha$ spécifiques de ligand de ces récepteurs permettrait, d'autre part, de rendre compte du caractère plus (HILDA/LIF, IL-6, OSM) ou moins (CNTF) pléiotrope de ces cytokines.

\section{Transduction du signal}

Les effets biologiques maximaux induits par l'HILDA/LIF, tout comme ceux induits par l'IL-6, sont obtenus à des taux faibles d'occupa- tion des récepteurs de haute affinité (1 à $10 \%$ ). C'est le cas de l'induction de la différenciation de la lignée $\mathrm{M} 1$, de la prolifération de la cellule DA1, ou de l'activité phosphatase alcaline de la cellule préostéoblastique RTC-1, ce qui suggère des mécanismes très efficaces de transduction liés à l'activation du récepteur. Les éléments moléculaires qui sous-tendent ces mécanismes sont actuellement inconnus. Bien que les sous-unités gp130 et gp190 ne contiennent pas de séquences consensus prédictives d'activités de type protéine kinase, il a été montré que l'activation du récepteur HILDA/LIF est accompagnée par la phosphorylation de nombreux substrats. Sur la lignée M1, l'HILDA/LIF entraîne la phosphorylation sur des résidus sérine/thréonine de la protéine de stress p27 et induit, tout comme l'IL-6, la phosphorylation sur des résidus tyrosine d'une protéine de $160 \mathrm{kDa}$ [21]. Une étude effectuée sur une lignée de cellules nerveuses de rat (MAH) a montré que l'HILDA/LIF et le CNTF, qui tout deux inhibent la prolifération de ces cellules, induisent de manière similaire la phosphorylation (tyrosine) de trois protéines majeures [20]. L'une d'elle (CLIP1) a été identifiée à la gp130, une deuxième (CLIP2) pourrait être la gp190, la troisième (CLIP3, $75 \mathrm{kDa}$ ) pouvant être une tyrosine kinase associée. Dans ce contexte, il a été récemment montré que l'OSM induit l'activation de la tyrosine kinase p62 ycs [22]. Ces études sont autant d'éléments qui confortent les analogies fonctionnelles des récep- 
Figure 3. Structure et arrangement des chaînes des récepteurs humains de I'HILDA/LIF, IL-6, OSM et du CNTF. A. CHR : région d'homologie des récepteurs des cytokines hématopoiétiques. Les barres représentent les résidus cystéines. La partie grisée WS représente le motif WSXWS. Ig: région conservée de la superfamille des immunoglobulines. $F N$ : domaine fibronectine de type III. EC, TM et CYT : domaines extracellulaire, transmembranaire et cytoplasmique. Les chiffres en regard indiquent le nombre d'acides aminés de chacun des domaines. B. Arrangement des chaînes gp 190, gp80 et gp130 (chaînes de basse affinité respectivement pour I'HILDA/LIF, I'IL-6 et I'OSM) et gp72 (récepteur CNTF) en structures de haute affinité pour ces différentes cytokines. (Adapté d'après [14, 20].) En pointillé sont indiquées les chấnes $\alpha$ potentielles des récepteurs HILDA/LIF et OSM.

teurs de l'HILDA/LIF, de IL-6, de OSM et du CNTF.

\section{Cellules productrices d'HILDA/LIF}

Les sources cellulaires d'HILDA/LIF (expression de l'activité biologique et/ou des transcrits) sont très variées (Tableau II). In vitro, l'HILDA a été initialement caractérisée dans le milieu conditionné de clones lymphocytaires $T$ issus d'un rein rejeté [2]. Elle est aussi sécrétée in vitro par les monocytes après stimulation par un ester de phorbol en association avec le 1-25 dihydroxycholécalciférol [8]. De nombreuses lignées leucémiques stimulées par des mitogènes produisent l'HILDA/LIF ; parmi elles la lignée T HTLV-I positive C10MJ2 à partir de laquelle l'ADNc de l'HILDA a été clonée [23], la lignée lymphoblastoïde $\mathrm{HSB} 2$, la lignée murine LB3 à partir de laquelle l'ADNc du LIF a été cloné [24] et les lignées monocytaires humaines U937, HL60 et THP1. Divers types de cellules non hématopoïétiques comme les cellules myocardiques de rat, les cellules de foie de rat Buffalo, et diverses lignées tumorales humaines (carcinomes, mélanomes, neuroblastomes) produisent constitutivement la cytokine in vitro [25].

$\mathrm{m} / \mathrm{s} n^{\circ} 9 \mathrm{vol} .8$, novembre 92

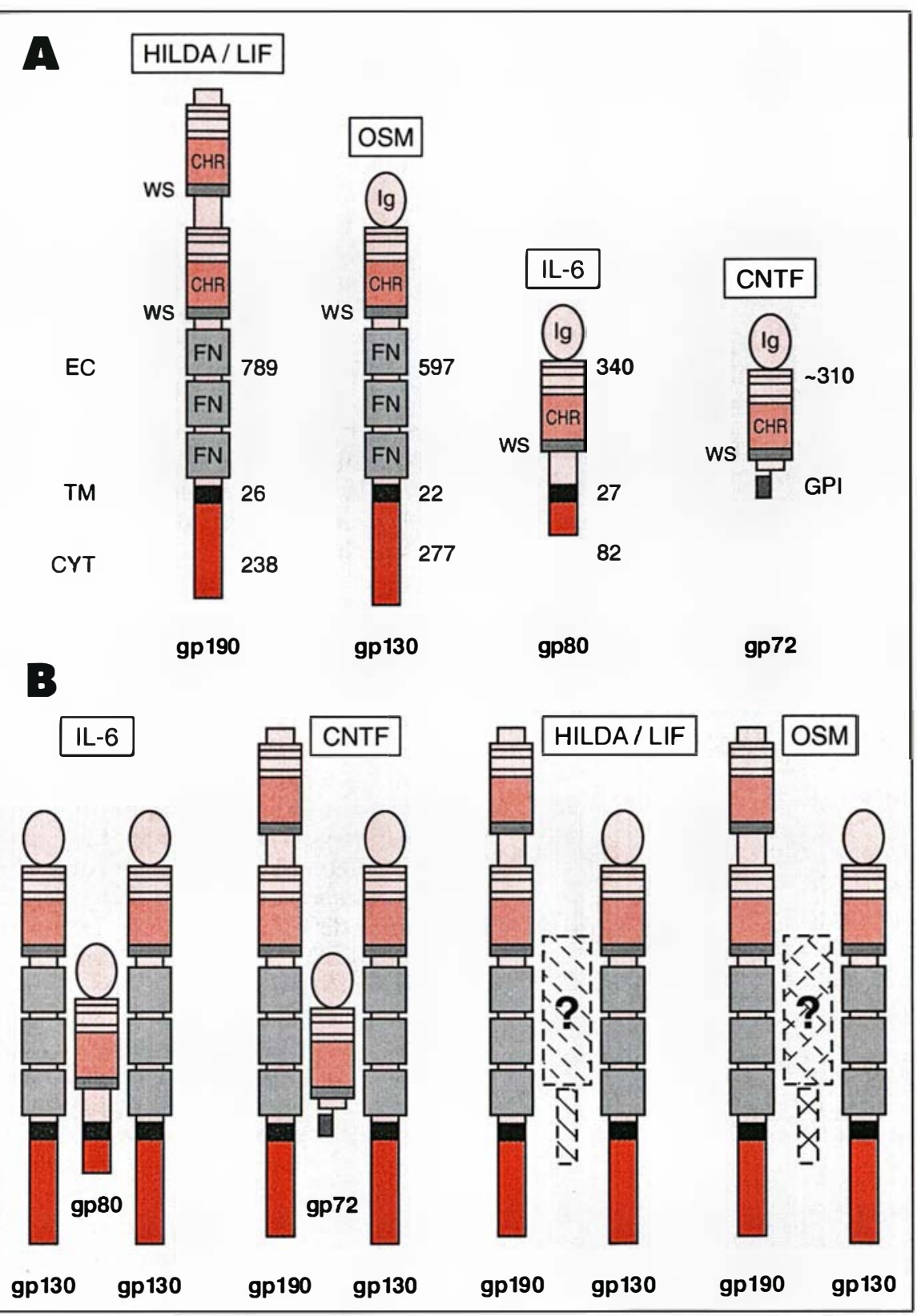

In vivo, l'HILDA/LIF a été détectée chez la souris nude greffée avec le mélanome SEKI [26] et dans les ascites murines de KrebsII et d'Ehrlich. Chez la souris embryonnaire ou adulte normale, un certain nombre de tissus exprime des transcrits HILDA/LIF mais à des niveaux très faibles (tissu calvaire, peau, intestin...), ce qui laisse supposer l'existence de mécanismes très efficaces de contrôle de cette expression. Aucune activité HILDA/LIF n'est détectée dans le sérum et sa demi-vie, lorsquelle est injecté par voie intravei- neuse, est très courte (quelques minutes) [27], ce qui indique que l'HILDA/LIF agit plutôt de manière localisée que par voie systémique.

L'expression la plus nette des transcrits HILDA/LIF est observée dans les glandes endométriales de l'utérus. Cette expression est très transitoire. Elle a lieu au $4^{\mathrm{e}}$ jour après la fécondation (post-coitum, p.c.) et précède toujours l'implantation utérine de l'embryon [28]. L'embryon à l'état de blastocyste exprime également des transcrits HILDA/LIF avant la nidation ( 3,5 jours p.c.) et jusqu'au jour 


\section{RÉFÉRENCES}

36. Smith AG, Ileath JK, Donaldson DD, et al. Inhibition of pluripotential embryonic stem cell differentiation by purified polypeptides. Nature $1988 ; 336$ : 688-90.

37. Koopman P, Cotton RGH. A factor produced by feeder cells which inhibits embryonal carcinoma cell differentiation. Characterization and partial purification. Exp Cell Res 1984; 154: 233-42.

38. Cosgrove D, Gray D, Dierich A, et al. Mice lacking MHC II molecules. Cell 1991 ; 66 : 1051-66.

39. Stewart CL, Kaspar P, Brunet LJ, et al. Blastocyst implantation depends on maternal expression of leukaemia inhibitory factor. Nature $1992 ; 359$ : 76-9.

40. Yamamori T, Fukada K, Aebersold R, $e t$ al. The cholinergic neuronal differentiation factor from heart cells is identical to leukemia inhibitory factor. Science 1989 ; 246: 1412-6.

41. Baumann HH, Wong GG. Hepatocytestimulating factor III shares structural and functional identity with leukemia-inhibitory factor. J Immunol 1989 ; 143 : 1163-67.

42. Abe T, Murakami M, Sato T, et al. Macrophage differentiation inducing factor from human monocytic cells is equivalent to murine leukaemia inhibitory factor. $J$ Biol Chem 1989 ; 264 : 8941-5.

43. Blancho G, Moreau JF, Anegon I, et al. HILDA/LIF is present in the urine of rejecting kidney graft recipients. Transplant Int $1992 ; 5: 57-8$.

44. Taupin JL, Morel D, Moreau JF, et al. HILDA/LIF urinary secretion during acute kidney rejection. Transplantation 1992 ; 53 : 655-8.

45. Alexander HR, Wong GGH, Doherty GM, et al. Differentiation Factor/Leukemia Inhibitory Factor protection against lethal endotoxemia in mice : synergistic effect with interleukin 1 and tumor necrosis factor. $J$ Exp Med $1992 ; 175$ : 1139-42.

46. Austin L, Burgess AW. Stimulation of myoblast proliferation in culture by leukaemia inhibitory factor and other cytokines.
12,5 p.c. [29]. La présence d'activité biologique HILDA/LIF est détectée dans les milieux conditionnés des blastocytes mis en culture in vitro.

La transcription du gène HILDA/LIF au niveau de l'embryon a lieu à 7,5 jours p.c. dans l'ectoderme extraembryonnaire et à $9,5,10,5$ et 13,5 jours p.c. dans le placenta [29]. A cette même période $(11,5$ et 13,5 jours p.c.), l'HILDA/LIF n'est pas transcrite dans l'embryon à proprement parler. Bien que la mise en culture de ces derniers permette d'obtenir des fibroblastes qui sécrètent la molécule, les cellules ES (embryonic stem cells) de la masse cellulaire interne de l'embryon ne sécrètent pas ou très peu d'HILDA/LIF in vitro.

\section{Activités biologiques}

\section{Hématopoï̀̀se}

Les premières actions décrites pour l'HILDA/LIF ont pour cible deux lignées murines d'origine hématopoiétique, la DA1a et la M1, avec des effets diamétralement opposés. Parmi une panoplie de cellules dépendantes de l'IL-3, la lignée DA1a est la seule à proliférer en réponse à l'HILDA/LIF [30]. Cette lignée répond cependant également au GCSF et à l'OSM humains. A l'opposé, l'HILDA/LIF a été aussi identifiée sous les noms de LIF [24] et de $D$-factor [1], respectivement, par sa propriété d'inhiber la prolifération et d'induire la différentiation macrophagique de la lignée leucémique murine M1. Le LIF induit une diminution de la taille et du nombre de colonies formées en agar par la lignée M1, probablement par une réduction du nombre des cellules clonogéniques dans la population cellulaire totale. Les effets ou les récepteurs de l'HILDA/LIF sur la cellule M1 ne sont pas retrouvés sur d'autres lignées leucémiques myéloïdes murines. Les mécanismes qui expliquent les effets opposés de l'HILDA/LIF sur les lignées DA1a et M1 sont inconnus, mais il faut remarquer que les deux lignées cellulaires montrent le même type de réponse en présence d'autres cytokines telles que le G-CSF.

Chez l'homme, l'HILDA/LIF inhibe la formation de colonies à partir des leucémies myéloïdes chroniques et prolonge les phases non $\mathrm{S}$ du cycle dans les cellules clonogéniques des leucémies myéloblastiques aiguës. A la différence des CSF et de l'IL-6, l'HILDA/LIF n'a aucun effet prolifératif sur les leucémies déjà testées. L'HILDA/LIF, en combinaison avec d'autres cytokines, inhibe faiblement le potentiel clonogénique des lignées leucémiques myélomonocytaires humaines U937 et HL-60. Ni la morphologie ni le phénotype de ces lignées ne sont modifiés par la présence de l'HILDA/LIF, seule ou en combinaison avec des cytokines ou des agents de différenciation tels que le 1,25-dihydroxycholécalciférol. L'HILDA/LIF est aussi capable de réduire, en combinaison avec le 1-b$\mathrm{D}$ arabinofuranosylcytosine, la prolifération clonogénique de la lignée U937, et de façon concomitante d'induire sa différenciation macrophagique.

L'HILDA/LIF, tout comme l'IL-6 et le G-CSF, est capable, en combinaison avec l'IL-3, d'induire la formation des colonies blastiques très indifférenciée à partir des progéniteurs hématopoïétiques humains [31]. L'effet synergique de l'IL-6 implique un passage plus rapide des cellules souches hématopoïétiques par le stade $G_{0}$. Un tel mécanisme reste à démontrer dans le cas de l'HILDA/LIF. L'HILDA/LIF augmente également la croissance et la prolifération des colonies des précurseurs plus tardifs, érythroïdes et éosinophiles. Une activation fonctionnelle des éosinophiles a été décrite [32], mais il est probable que l'effet observé avec la préparation purifiée à partir des surnageants de clones $\mathrm{T}$ soit dû à une cytokine contaminante qui pourrait être l'IL-5.

L'HILDA/LIF augmente l'efficacité d'infection rétrovirale in vitro des progéniteurs hématopoiétiques murins [33], effet qui pourrait être lié à l'activation des divisions cellulaires, dont on sait qu'elles sont nécessaires à l'intégration d'un provirus dans le génome d'une cellule hôte. Dans ce contexte, l'utilisation de l'HILDA/LIF doit pouvoir faciliter l'intégration d'ADN recombinant par des vecteurs rétroviraux, ce qui ouvre des perspectives intéressantes dans le domaine de la thérapie génique.

L'injection, chez la souris, de fortes 
doses d'HILDA/LIF recombinante entraîne de nombreux effets hématologiques [34]: stimulation de la mégacaryopoïèse avec une augmentation des mégacaryocytes (moelle osseuse, rate) et des plaquettes ; stimulation de l'érythropoïèse avec une augmentation des précurseurs érythrocytaires (moelle, rate) ; augmentation des granulocytes mûrs (moelle, rate); absence ou diminution des éosinophiles dans le péritoine (site d'injection de l'HILDA/LIF) ; disparition presque totale des thymocytes corticaux et diminution des lymphocytes de la rate; nombre de leucocytes périphériques inchangé ; accélération de la sédimentation érythrocytaire.

Un deuxième modèle expérimental (inoculation chez la souris d'une lignée produisant de fortes quantités d'HILDA/LIF recombinante) a confirmé la plupart de ces observations. Cependant, une différence notable par rapport au premier modèle est l'apparition d'un syndrome de myélofibrose caractérisé par un excès de formation de nouveau tissu osseux, une diminution consécutive des précurseurs hématopoïétiques dans la moelle osseuse couplée à l'apparition d'une hématopoïèse splénique [35].

\section{Activités sur les cellules souches embryonnaires et le développement embryonnaire}

Les cellules ES (embryonic stem cells), dérivés de la masse cellulaire interne d'un blastocyste avant son implantation, peuvent être maintenues en culture dans un état non différencié qui leur permet, après réintroduction dans un blastocyste receveur, de participer à la formation de toutes les lignées cellulaires somatiques et germinales de l'organisme adulte. Chez la souris, la conservation in vitro de cette totipotentialité nécessite la présence d'une couche nourricière de fibroblastes embryonnaires, ou la présence d'un facteur soluble (DIA ou DRF) [36, 37] produit par différents types cellulaires. Plusieurs arguments (sources cellulaires, caractéristiques biochimiques, compétition au niveau des récepteurs) ont indiqué que DIA/DRF est identique à l'HILDA/LIF [36]. Enfin, l'HILDA/ LIF recombinante purifiée, en l'absence de cellules nourricières, est capable de soutenir la prolifération de populations pures de cellules ES totipotentes. Des lignées de cellules ES murines ont été dérivées directement à partir du blastocyste en présence d'HILDA/LIF recombinante et en l'absence de fibroblastes. La présence de ces derniers permet néanmoins d'obtenir des taux de prolifération cellulaire plus importants. Les contributions respectives des formes soluble et matricielle de l'HILDA/LIF n'ont pas été évaluées, ainsi que l'effet d'autres cytokines. L'HILDA/LIF recombinante a permis de produire des clones ES dont certains gènes ont été inactivés par les techniques de recombinaison homologue. Ces clones ont, par la suite permis, après injection dans des blastocystes et réimplantation, d'obtenir des souris chimériques dans lesquelles l'expression des gènes mutés est abrogée [38].

L'accumulation d'ARNm pour l'HILDA/LIF et sa production par le blastocyste murin et par des cellules utérines et placentaires murines renforce la notion d'un rôle de l'HILDA/LIF in vivo dans le développement embryonnaire précoce. Des travaux récents ont partiellement conforté cet aspect [39]. Un modèle de souris transgéniques dans lesquelles le gène de l'HILDA/LIF a été délété par recombinaison homologue, a montré très clairement le rôle indispensable de l'HILDA/LIF dans l'implantation utérine de l'embryon. De manière inattendue, le développement embryonnaire n'est pas affecté dans ces souris, ce qui suggère qu' in vivo, d'autres facteurs sont capables de se substituer à l'HILDA/LIF pour assurer un développement embryonnaire normal $\left(\mathrm{m} / \mathrm{s} \quad n^{\circ} 8\right.$, vol. 8 , p. 873). Des cytokines comme l'OSM, l'IL-6 et le CNTF, dont les récepteurs utilisent des éléments de transduction communs avec celui de l'HILDA/LIF, sont des substituts potentiels.

Induction de la différenciation neuronale cholinergique

Le type de neurotransmetteur utilisé par les neurones dérivés de la crête neurale dans leur différenciation, noradrénergique ou cholinergique, est modifié in vitro, et probablement in vivo, par différents facteurs. L'un d'entre eux, le
CDNF, est identique à l'HILDA/LIF [40]. CDNF/HILDA/LIF induit les neurones sympathiques de rat à synthétiser de l'acétylcholine et plusieurs autres neurotransmetteurs, en inhibant la production de catécholamines. Ce contrôle phénotypique neuronal n'agit que sur la différenciation et n'affecte pas la prolifération ou la survie cellulaire. L'HILDA/LIF est aussi capable d'induire la production de vasoactive intestinal polypeptide (VIP). L'état d'hyperexcitabilité montré par les souris injectées avec des fortes concentrations d'HILDA/LIF pourrait être expliqué par des effets sur le système nerveux.

HILDA/LIF n'est pas la seule cytokine a être impliquée en même temps dans le contrôle de la différenciation et/ou prolifération hématopoïétique et de la différenciation neuronale. D'autres cytokines telles que IL-1, IL-2, IFN- $\gamma$ et IL-6 ont des effets divers sur différentes cellules du système nerveux et le nerve growth factor (NGF) a des effets sur l'hématopoïèse.

\section{Protéines de la phase aiguë de} l'inflammation

Les hépatocytes produisent une série de protéines plasmatiques (acute phase plasma proteins) en réponse à des réactions inflammatoires aiguës. Plusieurs cytokines sont en partie responsables du contrôle de leur production. Ainsi l'IL-1 règle préférentiellement la production de certaines de ces protéines (type 1 ; haptoglobine, $\alpha_{1}$-glycoprotéine acide, fraction C3 du complément) tandis que 1'IL-6 en contrôle d'autres (type II ; fibrinogène, $\alpha_{2}$-macroglobuline et thiostatine). Un troisième facteur, le HSF-III, induit le même groupe de protéines que l'IL-6 tout en étant différent de cette dernière. Plus récemment, sur la base de données fonctionnelles, immunologiques et structurales, il a été montré que l'HSF-III est identique à l'HILDA/LIF [41]. Comme pour l'IL-6, l'HILDA/LIF induit, dans des cellules d'hépatome humain, l'expression des gènes des acute phase proteins en agissant sur les régions promotrices de ces gènes. Néanmoins, la réponse globale hépatocytaire à l'IL-6 et à l'HILDA/LIF montre des différences qualitatives et quantitatives considérables, suggérant l'existence de 
mécanismes d'action hépatocytaire spécifiques de chaque cytokine. La production d'HILDA/LIF par des lymphocytes et des monocytes activés par divers stimuli suggère que l'HILDA/LIF pourrait jouer un rôle dans la réponse hépatocytaire pendant des réactions inflammatoires.

\section{Inhibition de l'enzyme lipoprotéine lipase}

La cachexie se développe dans un organisme soumis à une infection chronique ou porteur d'une tumeur. Les altérations du métabolisme lipidique ont un rôle central dans ce processus. L'enzyme lipoprotéine lipase (LPL), présente dans les adipocytes, permet l'incorporation des acides gras circulants dans le tissu adipeux. L'inhibition de la LPL entraîne ainsi une diminution de cette incorporation et un catabolisme des tissus adipeux. Plusieurs cytokines dont le TNF- $\alpha$, l'IFN- $\gamma$ ou l'IL- 1 , produites par l'hôte en réponse aux tumeurs, ont ce type d'action inhibitrice. Un facteur, appelé melanoma lipoprotein lipase-inhibiting protein (MLPLI), a été purifié à partir d'une lignée de mélanome inductrice de cachexie [26]. La séquence primaire de l'extrémité $\mathrm{N}$-terminale du MLPLI est identique a celle de l'HILDA/LIF murin. A la différence des autres cytokines inhibant l'enzyme LPL, l'HILDA/LIF est un produit des cellules tumorales. L'injection chez la souris de fortes concentrations d'HILDA/LIF entraîne une diminution significative du poids corporel, suggérant ainsi un rôle de l'HILDA/LIF dans le développement de la cachexie $[34,35]$.

Activités sur le métabolisme osseux L'homéostasie osseuse est un mécanisme finement réglé qui fait intervenir, d'une part, les ostéoclastes responsables de la résorption osseuse et, d'autre part, les ostéoblastes qui assurent la production de nouveau tissu osseux (ostéogenèse). De nombreux facteurs (PDGF, FGF, TGF- $\beta 1$ ), et en particulier des cytokines (IL-1, $\mathrm{TNF} \alpha$ et $\beta$, IFN), participent à cette régulation. Parmi eux, le DIF est capable d'augmenter la résorption osseuse in vitro. Ce facteur a été purifié et est identique à l'HILDA/LIF a été confirmée avec de l'HILDA/LIF recombinante et nécessite une production de prostaglandines. Des études complémentaires indiquent que la cible cellulaire de l'HILDA/LIF est l'ostéoblaste et non l'ostéoclaste. Les ostéoblastes expriment des récepteurs de l'HILDA/LIF et produisent l'inhibiteur de type I de l'activateur du plasminogène (PAI-1) en réponse à la cytokine alors que les ostéoclastes n'ont pas le récepteur et que l'HILDA/LIF n'a pas d'action sur leur prolifération ou sur leur activité. Il semble donc que l'HILDA/LIF ait une action directe sur l'activité de formation osseuse de l'ostéoblaste (la production d'HILDA/LIF par ces cellules suggérant un mécanisme d'action de type autocrine) et que son activité de résorption osseuse passe par une action indirecte des ostéoblastes sur les ostéoclastes. Cette double activité de l'HILDA/LIF est en accord avec les résultats obtenus in vivo. L'injection, chez la souris, de cellules productrices d'HILDA/LIF provoque à la fois l'apparition de foyers de calcification dans des sites musculaires ainsi qu'une augmentation importante du nombre des ostéoblastes médullaires et une formation accrue de tissu osseux [34, 35].

Réponse immune et inflammation Le rôle de l'HILDA/LIF dans la réponse immune est mal connu. Il est cependant suggéré par la présence de la cytokine dans les surnageants de culture mixte lymphocytaire et de clones alloréactifs stimulés par l'antigène et l'IL-2 [2]. La disponibilité d'anticorps monoclonaux bloquant l'interaction de la cytokine avec ses récepteurs devrait permettre de progresser dans ce domaine. Récemment, l'HILDA/LIF a été trouvée dans les urines de receveurs d'allogreffes présentant un rejet aigu de type cellulaire $[43,44]$. Les taux diminuent sous traitement par corticoïdes et ne sont plus détectables trois semaines après le rejet. La présence simultanée dans les tissus rejetés de lymphocytes activés, potentiellement producteurs d'HILDA/LIF, et de macrophages portant les récepteurs de haute affinité suggère ainsi un rôle de la cytokine dans la réaction de rejet. L'HILDA/LIF joue aussi un rôle, très probablement comparable à ceux du TNF $\alpha$ et de l'IL-1, dans l'inflammation et la résistance aux endotoxines. Il a été récemment démontré un effet protecteur de l'HILDA/LIF dans le choc endotoxinique chez la souris, effet fortement synergique avec l'IL-1 et le TNF $\alpha$ [45].

Ainsi, cette cytokine est-elle un bon exemple de cette pléiotropie d'effet et de cette redondance fonctionnelle partielle qui caractérisent les cytokines auxquelles il est, de ce fait, bien difficile d'assigner souvent un rôle particulier bien défini. Dans le cas de l'HILDA/LIF, par exemple, le contraste est frappant entre la multiplicité des effets physiologiques d'une hyperproduction et la modicité des conséquences d'une inactivation homozygote par knock-out du gène codant pour cette cytokine $\left(\mathrm{m} / \mathrm{s} n^{\circ} 8\right.$, vol. 8, p. 873)

\section{Summary}

HILDA/LIF and related cytokines : pleiotropic effects and functional redundancy

HILDA/LIF is a highly glycosylated, 180 residus polypeptide secreted as a soluble factor by many cell types including $\mathrm{T}$ lymphocytes and monocytes. As a result of an alternative exon splicing event, an extracellular matrix-associated form of HILDA/LIF has also been described in mouse embryonic fibroblasts and ES cells. HILDA/LIF acts on many cell types expressing high-affinity receptors for the cytokine and of ten with opposite biological effects. It can either induce (DA1a leukemic cell line, myoblasts, endothelial cells) or inhibit (M1 monocytic leukemia cells, neuronal cells) cell proliferation and either induce (M1 cells, megacaryocytes, platelets) or inhibit (embryonic stem cells) cell differentiation. Among these various activities, perhaps the most patent and specific to HILDA/LIF are its ability to maintain the pluripotentiality of embryonic stem cells and its major role in uterine implantation of the embryo. HILDA/LIF is therefore a striking example of a group of 


\section{Summary (suite)}

pleiotropic cytokines which includes IL-6, G-CSF and oncostatine $\mathrm{M}(\mathrm{OSM})$. In addition, there is a high redundancy in the actions of these factors, many of the biological functions excrted by one of them being shared by some of the others. Molecular data at the cytokine and receptor levels have recently merged which provide a basis for these pleiotropic and redundant properties. First, from secondary structure prediction analysis, HILDA/LIF appears to belong to an helical cytokine family, the prototype of which, is growth hormone $(\mathrm{GH})$ and which also comprises IL-6, OSM and GCSF. Primary sequence homologies have also been found among the members of this family, the most significant being between HILDA/LIF and OSM. Moreover, homologics at the level of gene organisation and localisation are found for HILDA/LIF and OSM which suggest that they might dcrive from a common ancestor gene. Second, it has been found that two common transducing components enter in the structures and functionalitics of high-affinity receptors for this group of cytokines. One of them is the IL-6-R gp130 converter which is also associated with the receptors for HILDA/LIF, OSM and CNTF. The other is the low-affinity gp190 HILDA/LIF receptor itself, which is also part of the receptor structures for OSM and CNTF. The use of a common transducing machinery could partly explain the redundancy of these cytokines. A third, shorter $\alpha$ chain, devoid of intracellular transducing element, would confer ligand specificity for these receptors. It is known as gp80 in the case of IL-6-R and as gp72 in the casc of CNTF-R. The more or less tissue-restricted expression of this $\alpha$ chain would be one element controlling the pleiotropy of the cytokine.

\section{TIRÉS A PART}

\section{Y. Jacques.}

$\mathrm{m} / \mathrm{s} n^{\circ} 9$ vol. 8 , novembre 92 